\title{
SinI modulates the activity of SinR, a developmental switch protein of Bacillus subtilis, by protein-protein interaction
}

\author{
Uma Bai, ${ }^{2}$ Ines Mandic-Mulec, ${ }^{1,2}$ and Issar Smith ${ }^{1,2}$ \\ ${ }^{1}$ Department of Microbiology, The Public Health Research Institute, New York, New York 10016 USA; ${ }^{2}$ Department of \\ Microbiology, New York University Medical Center, New York, New York 10016 USA
}

\begin{abstract}
SinR, a 111-amino-acid DNA-binding protein, is a pleiotropic regulator of several late growth processes in Bacillus subtilis. It acts as a developmental switch, positively regulating genes for competence and motility and repressing aprE and stage II sporulation genes. It is encoded by the second gene in a two gene operon, but previous results have also indicated that these two genes are differently regulated. We show in this discussion that the product of sinI, the first open reading frame (ORF) of this operon, interferes with the function of SinR. In vivo experiments have demonstrated that overexpression of $\operatorname{sinI}$ results in phenotypes that are observed in cells with a null mutation of $\sin R$. A chromosomal in-frame deletion of sinI gives rise to a phenotype associated with higher levels of SinR. Thus, SinI acts as an antagonist to SinR. In vitro experiments have shown that the interaction between these two proteins is a direct one. SinI prevents SinR from binding to its target sequence on $a p r E$, and the two proteins form a complex that can be immunoprecipitated with antibodies to either SinR or SinI.
\end{abstract}

[Key Words: Sporulation; post-translational control; protein-protein interaction]

Received August 10, 1992; revised version accepted October 28, 1992.

Nutrient depletion initiates a series of responses in the soil bacterium Bacillus subtilis that enables it to survive under adverse conditions. Among these responses are the synthesis and secretion of degradative enzymes and antibiotics, the development of motility and competence and, finally, the appearance of spores (Smith 1992). These processes have overlapping control mechanisms, but they can be differentiated on the basis of specific genetic and nutrient requirements; for example, glucose is essential for competence but represses sporulation. Several proteins affecting these pathways have been described (Smith 1993). SinR (formerly known as Sin), a DNA-binding protein, is one of these regulators of developmental processes that are initiated at the end of exponential growth, in response to nutrient depletion (Smith 1989). Previous work has shown that $\operatorname{SinR}$ is a repressor of $a p r E$, the gene coding for the secreted protease, subtilisin, and it also inhibits sporulation (Gaur et al. 1986, 1991). It has recently been shown that SinR exerts its repressive effects on sporulation immediately before stage II, by inhibiting the expression of spoIIA, spoIIE, and spoIIG (Mandic-Mulec et al. 1992). On the other hand, null mutations in the $\sin R$ gene prevent development of competence and motility and the production of growth-related autolysins (Gaur et al. 1986; Sekiguchi et al. 1988). It has been shown that its requirement for the development of motility is at the level of motility regu- lon gene expression (M. Predich, M. Zelic, and I. Smith, unpubl.). Thus, SinR acts as a developmental switch, controlling alternate developmental pathways. Given this role, the concentrations or function of this protein must be tightly regulated.

We have shown previously that levels of $\operatorname{SinR}$, as measured by immunoassay, actually increase at a time when its stage II negative target genes showed increased transcription (Mandic-Mulec et al. 1992). These results suggested that $\operatorname{Sin} R$ function may be regulated at a posttranslational level. The sin operon, controlled by two upstream promoters, $\mathrm{P} 1$ and $\mathrm{P} 2$, consists of two adjacent open reading frames (ORFs), the downstream $\sin R$ and ORF-1, now called sinI. Recent experiments have indicated that promoter $\mathrm{P} 3$, between $\sin I$ and $\sin R$ can also function in vivo. Studies with translational lacZ fusions of $\sin R$ and $\sin I$ have shown that these genes are regulated differently (Gaur et al. 1988). sinI is under developmental control, being expressed at low levels during vegetative growth and at high levels during sporulation. Its expression requires the essential early sporulation genes spoOA and spoOH, and it is repressed by glucose, which blocks the early stages of sporulation. On the other hand, $\sin R$ is expressed at a similar low level throughout growth and its expression increases only slightly at the end of exponential growth (Gaur et al. 1988; Mandic-Mulec et al. 1992). $\sin R$ gene activity is 
not affected by any of the above genetic or nutrient factors that control $\sin I$ expression. In addition, greater sporulation inhibition was observed when plasmids contained only the $\sin R$ gene rather than the entire $\sin$ operon. Finally, multicopy plasmids containing $\sin I$ (but with mutations in $\sin R$ ) caused the appearance of colonies that were rougher than wild type and resembled those in which the chromosomal sinR gene had been disrupted. These results indicated that $\sin I$ may be acting as an antagonist of $\operatorname{Sin} R$.

In vivo data presented in this discussion support this observation. In addition, we have overexpressed and purified SinI. We have demonstrated by functional SinR assays and immunoprecipitation experiments that SinI and SinR interact physically with each other and that a direct protein-protein association is involved in the control of SinR activity.

\section{Results}

As discussed, several lines of evidence indicated that SinR function during development may be regulated by SinI. To provide additional evidence for this hypothesis, two types of experiments were performed. We first overexpressed the sinI gene in vivo and measured several cellular processes that have been shown to be regulated by SinR. Second, we inactivated the chromosomal $\sin I$ gene and investigated the results of this mutation on the same SinR-dependent processes.

\section{Overexpression of $\sin$ is similar to disruption of sinR in the chromosome}

To test whether $\sin I$ was specifically responsible for the rough phenotype observed in cells carrying the sin operon (both $\sin I$ and $\sin R$ ) on multicopy plasmids, but with the $\sin R$ inactivated, we cloned $\sin I$ by itself on a multicopy plasmid, resulting in pIS256. IS1576, a strain carrying pIS256, sporulated well, but colonies were extremely rough. We examined the microscopic appearance of this strain after overnight growth in nutrient sporulation medium (NSM). The cells displayed an unusual aggregation in the form of long filamentous septate chains (Fig. 1C) when compared with wild-type cells, IS75 (Fig. 1A). Placing the sinI gene behind the inducible pSPAC promoter and adding IPTG to a strain containing this construct integrated into the chromosome (IS791) gave a similar phenotype (Fig. 1D). These septate chains were similar to those observed when $\sin R$ was inactivated in the chromosome, for example, IS720 (Fig. 1B). Unlike the wild-type strain, the cells shown in Figure 1, $\mathrm{B}-\mathrm{D}$, were nonmotile, and on plates, colonies were very rough.

We then measured the levels of competence in strains overexpressing $\sin I$, as $\sin R$ is required for competence (Gaur et al. 1986; Albano et al. 1987). There was a drastic reduction of competence in IS1576, carrying pIS256, of approximately three orders of magnitude, as measured by the number of transformants. Similar results were obtained with strain IS791, carrying an integrated $\operatorname{sinI}$ gene behind the PSPAC promoter, after IPTG induction
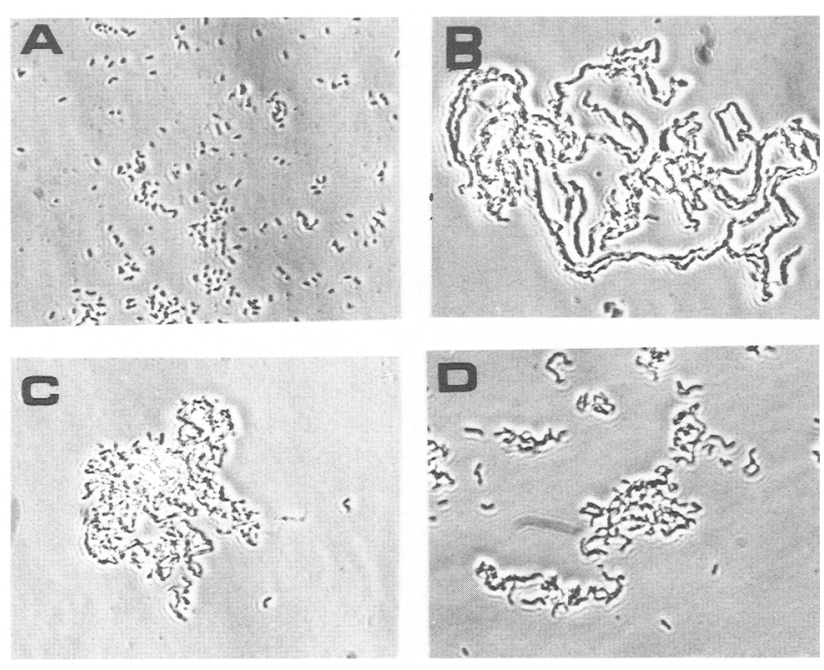

Figure 1. Photomicrograph of otherwise isogenic strains of $B$. subtilis in NSM. The cells were grown in NSM overnight at $32^{\circ} \mathrm{C}$ and were examined and photographed at $800 \times$ magnification, using phase-contrast optics (40× objective). (A) IS75 (wild type); (B) IS720 (chromosomal sinR null mutation); (C) IS1576 ( $\sin I$ on a multicopy plasmid); $(D)$ IS791 ( $\sin I$ behind an inducible pSPAC promoter integrated into the chromosome and induced with IPTG).

(data not shown). Because cells overexpressing $\sin I$ tend to aggregate, giving inaccurate viable counts, a more accurate measurement of competence was made by studying the effects of $\sin I$ overexpression on the late competence gene comG (Albano et al. 1987). pIS256 was introduced into a strain containing a comG-lacZ fusion (integrated at the $a m y E$ locus), and $\beta$-galactosidase-specific activity was measured by growth in liquid minimal salts competence medium. The results are shown in Figure 2. com $G$ expression was reduced approximately threefold in IS1593, carrying pIS256, as compared with IS1594, which carries pIS257, a plasmid with a deletion in $\sin I$.

We then investigated the effects of SinI overproduction on spoIIA, a stage II sporulation gene that is repressed by SinR (Mandic-Mulec et al. 1992). For this study we used IS820, a strain with a spoIIA-lacZ fusion and also carrying the $\sin I$ gene behind the pSPAC promoter, integrated into the chromosome. The expression of spoIIA was measured in IS820 after inducing the culture with IPTG. As shown in Figure 3, spoIIA was overexpressed at a level fourfold higher than wild-type cells (IS819). Similar quantitative results have been obtained with spoIIA-lacZ fusions when the chromosomal $\sin R$ gene was inactivated (Mandic-Mulec et al. 1992).

The above results, summarized in Table 1 , indicate that overexpression of $\sin I$ caused a pleiotropic phenotype resembling that observed when the chromosomal $\sin R$ gene was inactivated. The fact that these effects were observed both with multicopy $\sin I$ and with the $\sin I$ gene integrated into the chromosome behind an inducible promoter indicated that the $\sin I$ gene product was responsible for the observed phenotypes. 


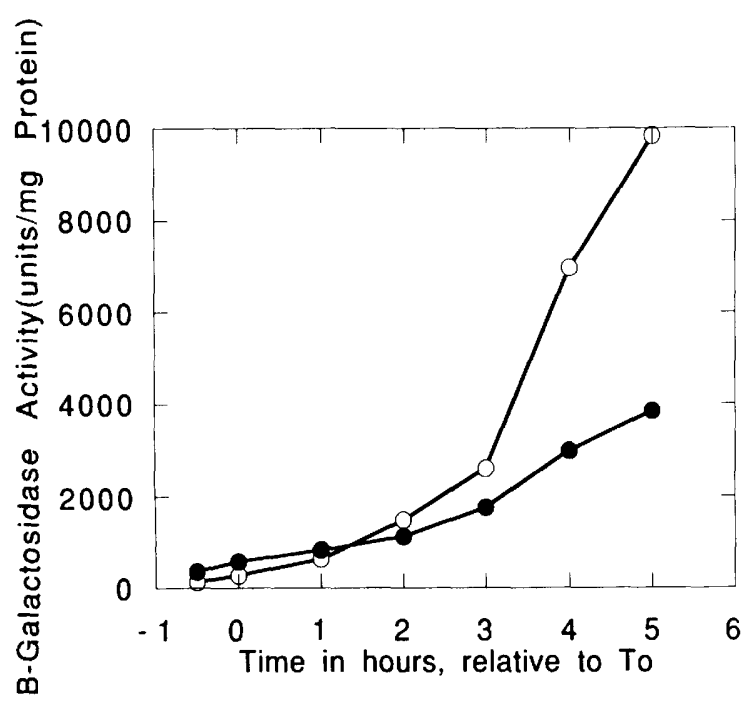

Figure 2. Effect of $\sin I$ on $\operatorname{com} G-1 a c Z$ expression. $\beta$-Gal was assayed in strains containing an integrated com $G-1 a c Z$ fusion (IS803) grown in minimal salts competence medium, and samples were withdrawn at indicated times. (O) IS1593 /carrying pIS256 with an intact sinI); (O) IS1594 (carrying pIS257 with a deletion in sinI $)$. $\left(\mathrm{T}_{0}\right)$ The time at which the cells entered the stationary phase of growth; $\left(T_{-1}\right)$ an hour before $T_{0} ;\left(T_{+1}\right)$ an hour after $\mathrm{T}_{0}$, etc.

\section{Inactivation of $\operatorname{sinl}$ results in a $\operatorname{Sin} R^{+}$phenotype}

Because overexpression of $\sin I$ resulted in a phenotype similar to that observed in chromosomal $\sin R$ null mutants, we wanted to examine the effect of a sinI chromosomal deletion. According to our hypothesis about the role of SinI, it was predicted that cells with a disrupted $\sin I$ gene would behave as though $\operatorname{Sin} R$ were overpro-

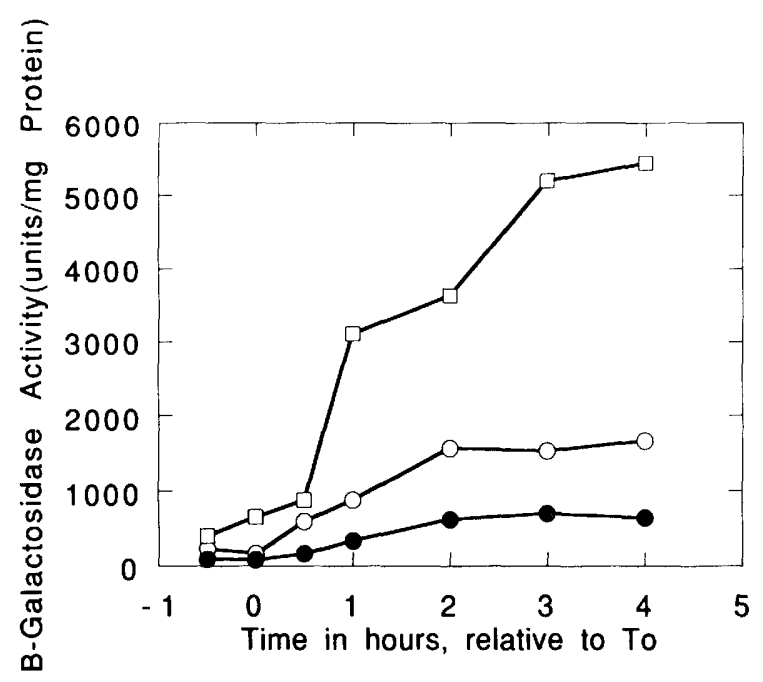

Figure 3. Effect of $\sin I$ on spoIIA-lacZ expression. Expression of $\operatorname{spoIIA}$ was assayed by measuring $\beta$-gal activity of spoIIAlac $Z$ fusions, in otherwise isogenic strains, during growth in NSM. (口) IS820 ( $\operatorname{sinI}$ behind a pSPAC promoter and induced with IPTG); (O) IS819 (wild type); (O) IS879 (with an sinI chromosomal null mutation). IPTG was added when the culture was inoculated.
Table 1. Comparison of phenotypic effects of sinI overexpression and $\sin \mathrm{R}$ deletions

\begin{tabular}{llc}
\hline & $\begin{array}{l}\text { Overexpression } \\
\text { of } \operatorname{sinI}\end{array}$ & $\begin{array}{c}\text { sinR deletion in } \\
\text { the chromosome }\end{array}$ \\
\hline $\begin{array}{c}\text { Colonial } \\
\text { morphology }\end{array}$ & rough & rough \\
$\begin{array}{l}\text { Sporulation } \\
\text { Competence }\end{array}$ & $\begin{array}{c}\text { spoIIA: fourfold } \\
\text { overexpressed }\end{array}$ & $\begin{array}{c}\text { spoIIA: fourfold } \\
\text { overexpressed }\end{array}$ \\
& decreased & 3 log orders \\
& comG expression & decreased \\
& reduced & reduced \\
Motility & nonmotile & nonmotile \\
Microscopic & long filamentous & long filamentous \\
examination & chains with & chains with \\
& septations & septations \\
\hline
\end{tabular}

duced (a SinR ${ }^{+}$phenotype). A nonpolar, in-frame deletion was made by deleting 33 codons from the center of the sinI ORF in pIS256, creating pIS257. This plasmidborne $\sin I$ deletion in pIS257 was then integrated into the chromosome by gene conversion, as described in $\mathrm{Ma}$ terials and methods. The resulting strain, IS874, had a smooth translucent colonial morphology, and its sporulation was reduced to $1-10 \%$ of wild-type levels. Similar sporulation results were obtained when the chromosomal $\sin I$ was disrupted with a kanamycin resistance cassette (data not shown). Because, as shown above, $\sin I$ activated the expression of spoIIA fourfold, we asked whether a sinI deletion would cause lowered spoIIA expression. The results are shown in Figure 3. spollA-lacZ activity was repressed in IS879, carrying the in-frame deletion of $\sin I$ in the chromosome, compared with a wild-type strain (IS819).

We have shown previously that overproduction of SinR inhibits the production of extracellular proteases (Gaur et al. 1986). We assayed the production of extracellular protease activity in a strain carrying an inactivated $\sin I$ gene, IS 880 . In the same experiment, for comparison, we also measured extracellular protease activity in a strain carrying the $\sin R$ gene on a multicopy plasmid (IS1491). Very little exoprotease activity was observed in these strains compared with the amounts observed in a control strain (IS812) (Fig. 4).

Thus, the cells with disrupted $\sin I$ behaved as though SinR were overproduced. The results are summarized in Table 2. This Sin $R^{+}$phenotype observed in strains with a deletion of $\sin I$ could be eliminated by reintegrating a wild-type $\sin I$ at the region of homology, providing SinI in trans on a multicopy plasmid, or by inducing the strain carrying the pSPAC sinI construct. This indicated that the phenotype was caused by the disruption in $\sin I$.

\section{SinR levels are not significantly affected by sinI expression}

The above results clearly indicate that SinI controlled the activity of $\sin R$, but the nature of this interaction was unknown. It was possible that $\operatorname{SinI}$ modulated $\sin R$ 


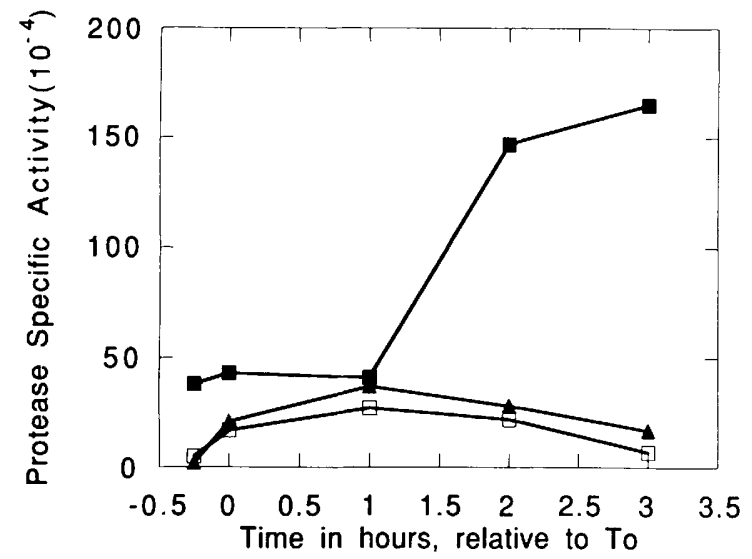

Figure 4. Effect of sinI disnuption on extracellular protease production. Cells were grown in NSM, and extracellular protease activity was measured in cell-free supernatants of IS657 la wild-type strain) ( $\square$ ); in IS1491 (carrying $\sin R$ on a multicopy plasmid) ( $\square$ ); and in IS880, having a deletion of $\sin I$ in the chromosome ( $\mathbf{A}$ ). Activity is expressed in units, as described in $\mathrm{Ma}$ terials and methods.

gene expression or the stability of the SinR protein, which could explain the physiological data described above. To see whether overexpression of $\sin I$ or its absence alters cellular SinR levels, we measured SinR concentrations during growth in strains overexpressing $\sin I$ or in strains with a $\sin I$ deletion.

Cultures of IS1576, harboring a multicopy plasmid with sinI, and IS1556, a wild-type strain carrying a plasmid vector, were grown in NSM, and samples were withdrawn at regular intervals for immunodetection of SinR using polyclonal antibodies raised against the SinR protein. The results are shown in Figure 5A. The levels of SinR increased slightly during growth in both of these strains. Overexpression of $\sin I$ did not cause a reduction in the levels of SinR. The levels of SinR were actually higher in the strain overexpressing $\sin I$. This effect presumably reflects the autogenous control of the sin operon by SinR (Smith et al. 1991; N. Gaur and I. MandicMulec, unpubl.). A similar experiment was performed

Table 2. Comparison of phenotypic effects of sinI deletions and $\sin \mathrm{R}$ overexpression

\begin{tabular}{lll}
\hline & $\begin{array}{l}\text { sinI deletion in } \\
\text { the chromosome }\end{array}$ & $\begin{array}{l}\text { Overexpression } \\
\text { of } \sin R\end{array}$ \\
\hline $\begin{array}{c}\text { Colonial } \\
\text { morphology }\end{array}$ & smooth & smooth \\
$\begin{array}{lll}\text { Phenotype } \\
\text { Spo }^{-}(1-10 \% \\
\text { of wild type }) \\
\text { spoIIA expression } \\
\text { inhibited }\end{array}$ & $\begin{array}{c}\text { Spo }^{-}(2-3 \text { orders of } \\
\text { magnitude decrease }) \\
\text { spoIIA expression } \\
\text { inhibited }\end{array}$ \\
$\begin{array}{l}\text { Competence } \\
\text { Motility }\end{array}$ & $\begin{array}{l}\text { competent } \\
\text { normal } \\
\text { Extracellular } \\
\text { protease }\end{array}$ & $\begin{array}{l}\text { competent } \\
\text { normal }\end{array}$ \\
\hline
\end{tabular}
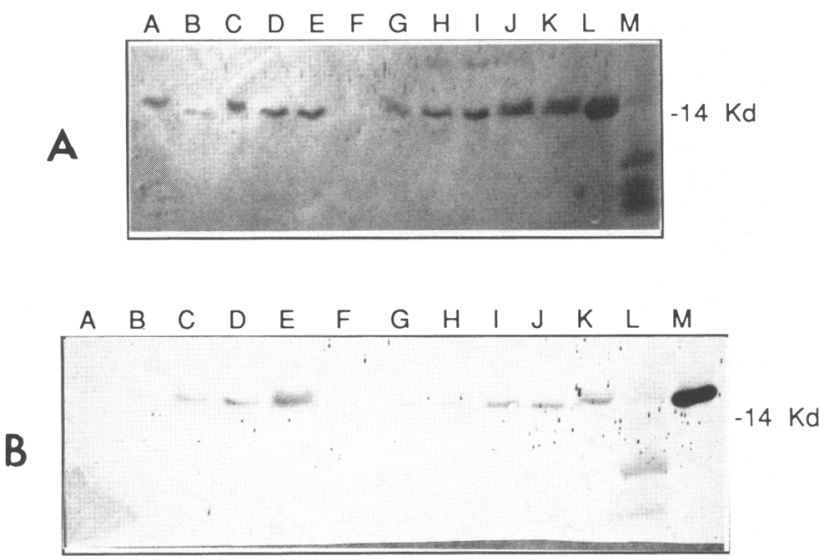

Figure 5. Effect of $\sin I$ on $\operatorname{SinR}$ protein levels during growth. The strains were grown in NSM, and cell-free extracts were prepared and subjected to Western blot analysis as described in Materials and methods. $(A)$ (Lanes $A-E$ ) SinR levels in IS1556, carrying vector plasmid pIS253, at times $\mathrm{T}_{-0.25}, \mathrm{~T}_{0}, \mathrm{~T}_{1}, \mathrm{~T}_{2}$, and $\mathrm{T}_{3}$, respectively. (Lane $F$ ) IS432, carrying a chromosomal deletion of $\sin R$, at $\mathrm{T}_{2}$. (Lanes $G-K$ ) SinR levels in IS1576, carrying $\sin I$ on a multicopy plasmid pIS256, at times $\mathrm{T}_{-0.25}, \mathrm{~T}_{0}, \mathrm{~T}_{1}, \mathrm{~T}_{2}$, and $\mathrm{T}_{3}$, respectively. (Lane $L$ ) Purified $\operatorname{SinR}$ used as a positive control. (Lane $M$ ) Molecular mass standards. $(B)$ (Lanes $A-E$ ) SinR levels in IS1595, a control strain, at $\mathrm{T}_{-0.25}, \mathrm{~T}_{0}, \mathrm{~T}_{1}, \mathrm{~T}_{2}$, and $\mathrm{T}_{3}$, respectively. (Lane $F$ ) IS432 at $\mathrm{T}_{2}$. (Lanes $G-K$ ) SinR levels in IS874, carrying an inactivated sinI in the chromosome, at $\mathrm{T}_{-}{ }^{0.25}, \mathrm{~T}_{0}, \mathrm{~T}_{1}, \mathrm{~T}_{2}$, and $\mathrm{T}_{3}$, respectively. (Lane $L$ Molecular mass standards. (Lane $M$ ) Purified SinR protein.

with IS874, a strain carrying a deletion of $\sin I$ in the chromosome, and a control strain, IS1595; the results are shown in Figure 5B. A deletion of $\sin I$ did not result in higher levels of SinR in vivo. Thus, the effects of $\sin I$ overexpression or absence could not be explained by the alteration of SinR levels.

\section{SinI inhibits DNA binding of $\operatorname{Sin} R$ to aprE}

The finding that SinR levels are not affected significantly by overexpression or absence of $\sin I$ clearly indicated that $\sin I$ is able to regulate the activity of $\operatorname{Sin} R$ at a post-translational step. To investigate the nature of this mechanism, we wanted to study interactions between SinR and SinI in vitro. We first overproduced SinI in Escherichia coli, using a bacteriophage T7 RNA polymerase-controlled system (Studier et al. 1990). We were able to purify SinI in one step, taking advantage of its low binding to a DEAE-Sephacel column at $\mathrm{pH} 8.2$ (Fig. 6). We observed a protein of $\sim 6 \mathrm{kD}$, which is consistent with the predicted molecular mass from the derived amino acid sequence. Interestingly, SinI also did not bind to DNA affinity columns used to purify DNA-binding proteins (Fig. 7; data not shown). The purified protein reacted with the antibody raised against a synthetic polypeptide containing the middle 33 amino acids of SinI. Furthermore, the amino acid sequence of the first 20 amino acids of the purified protein, as determined by 


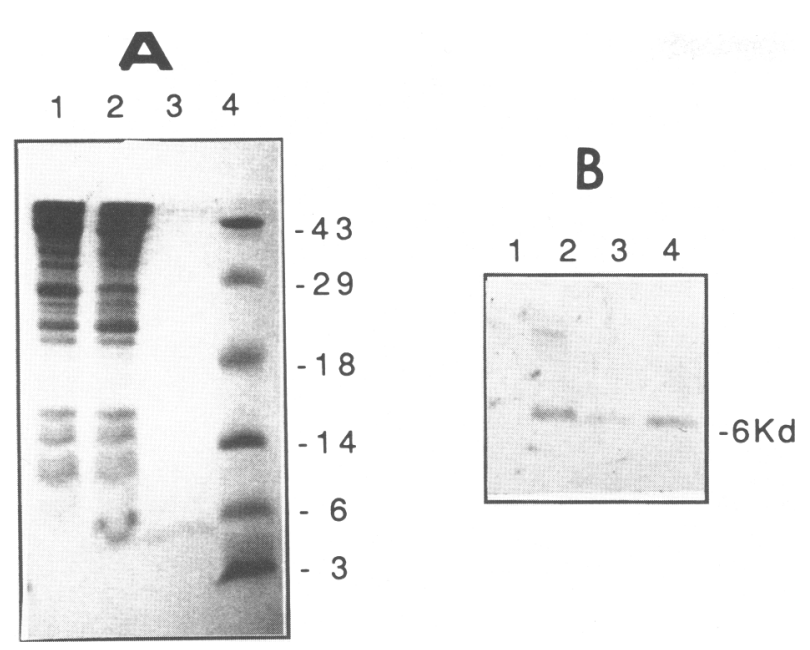

Figure 6. $(A)$ Purification of SinI. Samples from the purification steps were subjected to electrophoresis on a $12 \%$ SDSpolyacrylamide gel, and the gel was stained with Coomassie blue. (Lane 1) Fifty micrograms of crude extract of $E$. coli BL21(DE3)pLysS strain, harboring the vector pT7-1, after IPTG addition. (Lane 2) Fifty micrograms of crude extract of the above strain, but carrying the sinI-containing plasmid, pIS281, after IPTG addition. (Lane 3) Three micrograms of SinI from pooled DEAE-Sephacel fractions, containing SinI. (Lane 4) Protein standards. The numbers at left indicate the migration (in $\mathrm{kD}$ ) of these standards. (B) Immunochemical identification of SinI. Proteins from the extracts, as described above, were separated on a $12 \%$ SDS-polyacrylamide gel and transferred onto a nitrocellulose filter that was analyzed by immunoblotting with antiserum against SinI. The molecular mass of the protein is seen at right. (Lane 1) Fifty micrograms of crude extract of $E$. coli BL21(DE3)pLysS, containing pT7-1. (Lane 2) Fifty micrograms of crude extract of $B L 21(D E 3) \mathrm{p} L y S S$ containing pIS281, after IPTG induction. (Lanes 3 and 4) Fractions from DEAE-Sephacel column of extract illustrated in lane 2.

micro-Edman degradation, was identical to that predicted from the SinI ORF (data not shown). To study the nature of the reaction between SinR and SinI, we then tested the effect of SinI on the binding of purified SinR (Gaur et al. 1991) to its target sequence on aprE, in a gel mobility-shift assay.

SinR binds to aprE in a region $220-260$ bp upstream from the transcription start site (Gaur et al. 1991). In the studies reported here we used as a probe a 250-bp fragment of the aprE promoter and upstream regulatory sequences. Different amounts of SinI were incubated at room temperature for 15 min with constant levels of SinR. The mixtures were then used for the gel retardation studies, which were performed as described previously (Gaur et al. 1991). SinI prevented SinR from binding to aprE in a concentration-dependent manner (Fig. 7, lanes 4-9). Complete inhibition of SinR binding was observed in the presence of $0.5 \mu \mathrm{M}$ SinI, which corresponds to a molar ratio of $1: 2$ monomers of SinR/SinI. However, it is impossible to discuss the true stoichiometry of the interaction because SinR behaves in solution as a tetramer, and the actual state of multimerization of SinI as purified is currently unknown. In addition, we have no estimate of the fraction of active molecules in each preparation. If SinR were allowed to bind to aprE / $15 \mathrm{~min}$ at room temperature) and were then challenged with 0.5 $\mu \mathrm{M}$ SinI at room temperature for $15 \mathrm{~min}$, the retarded band could still be seen (lane 10). Thus, SinI is much less able to inhibit the binding of $\operatorname{SinR}$ to aprE once the SinR-DNA complex is formed. SinI, by itself, does not bind to the aprE probe (lane 2). These results suggest that SinI can associate with SinR by protein-protein interaction and thus attenuates its ability to bind to DNA. Control gel retardation experiments with the ArgR protein and DNA containing "arg boxes" showed that SinI had no effect on the interaction of this regulatory protein and its DNA-binding sites (data not shown).

\section{SinI binds to $\operatorname{Sin} R$}

The results of the aprE-binding studies presented in the previous section suggested that SinI regulates SinR function by direct protein-protein interaction. To see whether the two proteins formed a complex, we performed immunoprecipitation experiments. [ ${ }^{35} \mathrm{~S}$ ]Methionine-labeled SinI was synthesized in vitro using a bacterial cell-free-coupled transcription translation system. We observed a protein of $6 \mathrm{kD}$ in the extract programmed with the SinI-containing plasmid pIS281 (Fig. 8, lane 2). This protein was immunoprecipitated with SinI-specific antibodies (Fig. 8, lane 7). The radiolabeled SinI was added to SinR, and the complex was mixed with the same SinR antibody used for the experiments described in Figure 5 . The resulting complexes were purified by binding to protein A-Sepharose beads and were then analyzed on SDS-polyacrylamide gels. The results of these experiments indicated that the SinR antibody could precipitate SinI in the presence of SinR (Fig. 8, lane 8) but not if SinR was absent (Fig. 8, lane 6). The addition of an

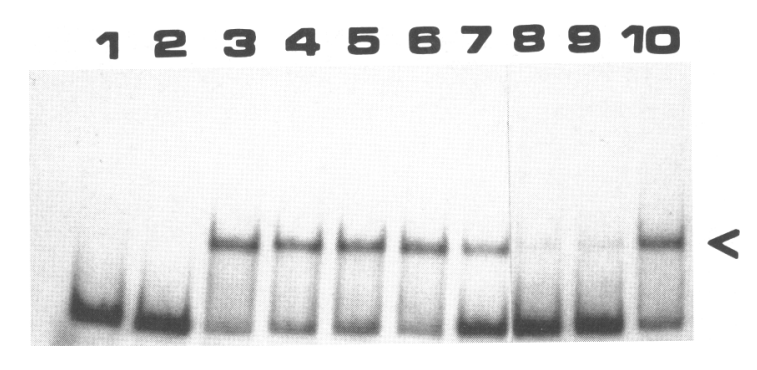

Figure 7. Effect of SinI on SinR binding to aprE. The $3^{\prime}$ endlabeled 250-bp DdeI-NotI fragment of aprE containing the SinRbinding region was used as a probe. In all lanes where SinR was present, its concentration was $250 \mathrm{nM}$. (Lane 1) Free probe; (lane 2) probe $+\operatorname{SinI}(500 \mathrm{nM})$; (lane 3) probe + SinR; (lane 4) probe $+\operatorname{SinR} / \operatorname{SinI}(15.63 \mathrm{nM})_{;}($lane 5$)$ probe $+\operatorname{SinR} / \operatorname{SinI}(31.25$ nM); (lane 6) probe $+\operatorname{SinR} / \operatorname{SinI}(62.5 \mathrm{nM}) ;$ (lane 7) probe $+\operatorname{SinR} / \operatorname{SinI}(125 \mathrm{nM}):($ lane 8$)$ probe $+\operatorname{SinR} / \operatorname{SinI}(250$ nM); (lane 9) probe $+\operatorname{SinR} / \operatorname{SinI}\left(\begin{array}{lll}500 & \mathrm{nM}\end{array}\right) ;$ (lane 10) probe $+\operatorname{SinR}$ for $15 \mathrm{~min}$ at room temperature. SinI was then added to a final concentration of $500 \mathrm{nM}$. The retarded band is indicated by the open arrowhead. 


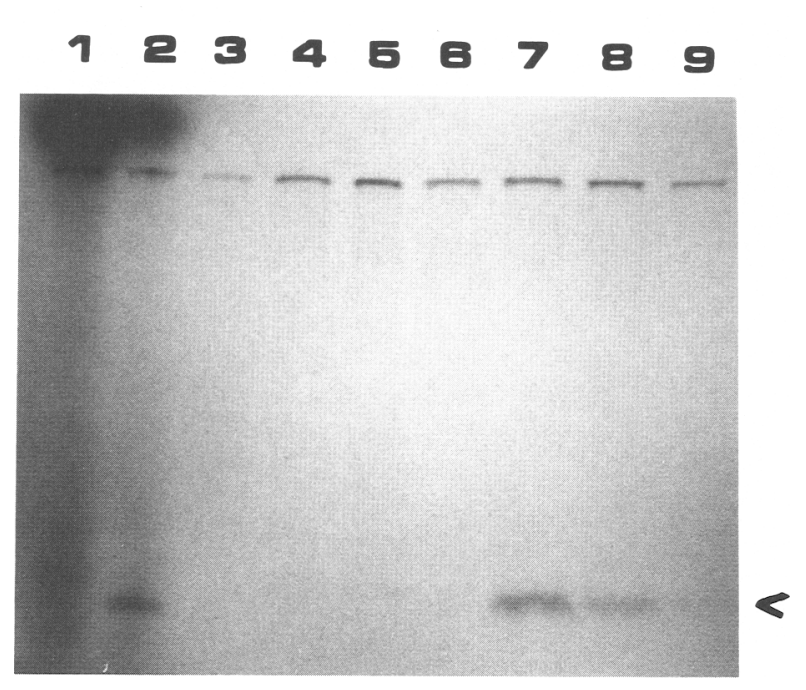

Figure 8. Coimmunoprecipitation of $\operatorname{SinI}$ and $\operatorname{SinR} .{ }^{35} \mathrm{~S}-\mathrm{La}-$ beled SinI was prepared using an S-30 extract of E. coli, as described in Materials and methods. Various samples, as described below, were fractionated on a $15 \%$ SDS-polyacrylamide gel followed by autoradiography. (Lane 1) In vitro-translated proteins of the vector pT7-1; (lane 2) ${ }^{35} \mathrm{~S}$-labeled SinI that was translated in the S-30 lysate carrying pIS281; (lane 3) in vitro-translated proteins of extracts containing pT7-1, subjected to immunoprecipitation with SinI antibodies; (lane 4) identical to lane 3, but using $\operatorname{SinR}$ antibodies; (lane 5) identical to lane 3, but SinR was added along with SinR antibodies; (lane 6) $\left[{ }^{35} \mathrm{~S}\right]$ methionine-labeled extracts containing pIS281, subjected to immunoprecipitation with SinR antibodies; (lane 7) identical to lane 6, but treated with SinI antibodies; (lane 8 ) identical to lane 6 but treated with SinR and SinR antibodies; (lane 9) identical to lane 8 but $1 \mu \mathrm{g}$ of nonradioactive SinI was added before adding SinR and SinR antibodies. The open arrowhead indicates the position of a standard molecular mass protein that migrated to a position corresponding to $\sim 6 \mathrm{kD}$.

excess of nonradioactive SinI to an immunoprecipitation reaction with $\operatorname{SinR}$ and $\operatorname{Sin} R$ antibodies decreased the amount of radioactivity observed in the SinI band (cf. lanes 8 and 9|, indicating that the labeled protein is SinI. The complementary experiment, using radioactive SinR, could not be performed because of difficulties in radiolabeling this protein in vitro. However, we were able to immunoprecipitate purified SinR with SinI antibodies in the presence of $\operatorname{SinI}$ as detected by Western immunoblotting (Fig. 9). We conclude that the SinR and SinI proteins bind directly to each other.

\section{Discussion}

SinR is a dual function regulatory protein exerting positive and negative effects on gene expression during $B$. subtilis late growth. It is needed for development of competence and motility and for the production of autolysin (Gaur et al. 1986; Sekiguchi et al. 1988). An increased expression of the $\sin R$ gene is inhibitory for sporulation and the production of extracellular proteases (Gaur et al. 1991; Mandic-Mulec et al. 1992). Thus, the function of the protein has to be turned off for cells to sporulate. How is this achieved? It seems likely that in addition to complex transcriptional regulation (Gaur et al. 1988), the activity of $\operatorname{Sin} R$ is modulated by SinI.

The in vivo results presented here indicate that $\sin I$ down-regulates $\operatorname{SinR}$ activity. Thus, when $\sin I$ was overexpressed, whether by being present on a multicopy plasmid or by means of an inducible promoter upstream from $\sin I$, SinR functions, such as the development of competence and motility as well as the repression of stage II-specific sporulation genes, were inhibited. Similarly, the phenotypes associated with elevated levels of SinR, such as the inhibition of stage II-specific gene expression and lowered production of extracellular proteases, are observed when $\sin I$ is inactivated in the chromosome. Thus, we have demonstrated that these two factors are functionally antagonistic. In addition, we have shown biochemically that SinI and SinR interact directly with each other. This is corroborated by the fact that SinI prevents SinR from binding to its target site on the aprE promoter (Fig. 7) and also that SinR antibody can immunoprecipitate SinI when the latter protein is mixed with SinR (Fig. 8). Conversely, SinR is immunoprecipitated by antibodies to SinI when mixed with SinI (Fig. 9).

What role does SinI play in normally differentiating $B$. subtilis? sinI is under sporulation control, its expression increasing with the onset of sporulation, and this rise in expression is inhibited by mutations in $s p o O H$ and spoOA. The former codes for a $\sigma$-factor, $\sigma^{\mathrm{H}}$ (Dubnau et

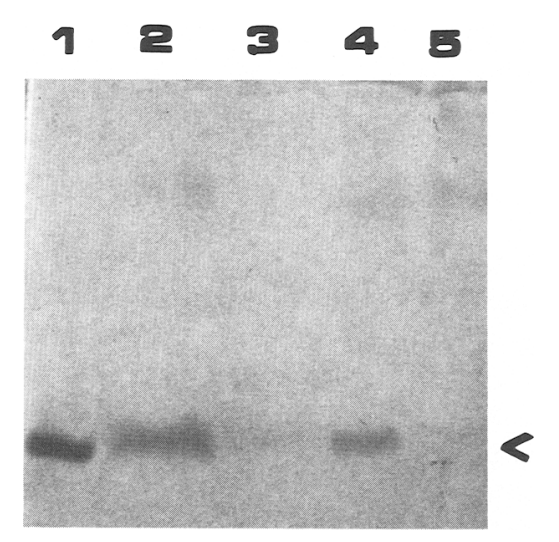

Figure 9. Coimmunoprecipitation of SinI and SinR. Purified SinR was treated in various ways, described below, and then immunoprecipitated (lanes 2-5) with SinI or SinR antibodies. The precipitates were fractionated by $15 \%$ SDS-PAGE, and the proteins were transferred to nitrocellulose papers that were probed with SinR-specific antibodies. (Lane 1) One microgram of purified SinR was added directly to the gel before electrophoresis; (lane 2) purified SinR immunoprecipitated with SinR-specific antisera; (lane 3) SinR immunoprecipitated with SinI-specific antisera; (lane 4) SinR mixed with purified SinI and immunoprecipitated with SinI-specific antisera; (lane 5) SinI immunoprecipitated with SinI antibodies. The open arrowhead indicates the position of a standard molecular mass protein corresponding to $\sim 14 \mathrm{kD}$. 
al. 1988; Zuber et al. 1989), which is essential for the transcription of essential early sporulation genes, including spoOA (Predich et al. 1992) and spoIIA (Wu et al. 1991). SpoOA is the key response regulator in the sporulation signal transduction pathway (Trach et al. 1992). Glucose, a catabolite inhibitor of sporulation, also represses $\sin I$ expression. $\sin R$ expression is unaffected by the genetic lesions and nutrient conditions described above (Gaur et al. 1986). This indicates that $\sin R$ is regulated differently than $\sin I$. $\sin R$ is transcribed by the $\mathrm{P} 3$ promoter, which is downstream from sinI. Insertion of an antibiotic resistance cassette into $\sin I$ does not prevent $\sin R$ expression, which is in agreement with the above conclusion. Thus, it can be postulated that $\sin I$ acts as a mediator between stage $\mathrm{O}$ and stage II sporulation genes that are repressed by SinR and that the information flows from spoOA to $\sin I$. During sporulation, SinR is down-regulated by elevated expression of $\sin I$, and the formation of the SinI/SinR complex may be one of the steps necessary to initiate or maintain the sporulation process. Because strains containing a deletion of both $\sin I$ and $\sin R$ have a phenotype identical to those with only the $\sin R$ gene inactivated, we believe that the sole function of SinI is to modulate SinR function (U. Bai, I. Mandic-Mulec, and I. Smith, unpubl.).

What is the mechanism by which SinI represses SinR activity? SinR is a DNA-binding protein (Gaur et al. 1986). Its amino-terminal region, which contains a putative helix-turn-helix (HTH) domain at positions 17-36, has a high degree of similarity to the amino terminus of the bacteriophage $\phi 105 \mathrm{R}$ protein (Smith et al.). Extensive mutational analysis has shown that this region of a \$105R contains a HTH DNA-binding domain (Van Kaer et al. 1988), and the similarity of the two proteins at their amino termini strongly suggests that this region is also the DNA-binding domain of SinR. Further analysis of the derived amino acid sequence of SinR revealed a series of leucine and other hydrophobic amino acids in heptad repeats following the putative DNA-binding domain, starting at position 47 and ending at position 68 . These data suggested the presence of an amphipathic coil structure in this region, which was postulated to be the domain for SinR multimerization (Gaur et al. 1991). The speculative $\operatorname{SinR}$ structure is presented in schematic form in Figure 10A (top).

Data base analysis of SinI has revealed that of all the several thousand proteins screened, SinI shows the greatest similarity to SinR. As shown in Figure 10A, in a 36-amino-acid overlap which includes the carboxy-terminal region of SinR and most of the 57-codon $\sin I$ ORF, there is a $28 \%$ identity and an additional $20 \%$ conservation of amino acids. Thus, SinI is missing the postulated DNA-binding domain of SinR; as shown in Results, SinI does not bind to DNA. A possible model of SinI function is suggested by the amino acid similarity to the carboxyterminal domain of SinR, its inability to shift DNA containing the aprE promoter, and its ability to form immunoprecipitable complexes with $\operatorname{SinR}$. Because $\operatorname{SinR}$, in solution, behaves as a tetramer, SinI may displace SinR monomers from SinR homomultimers to form hetero-

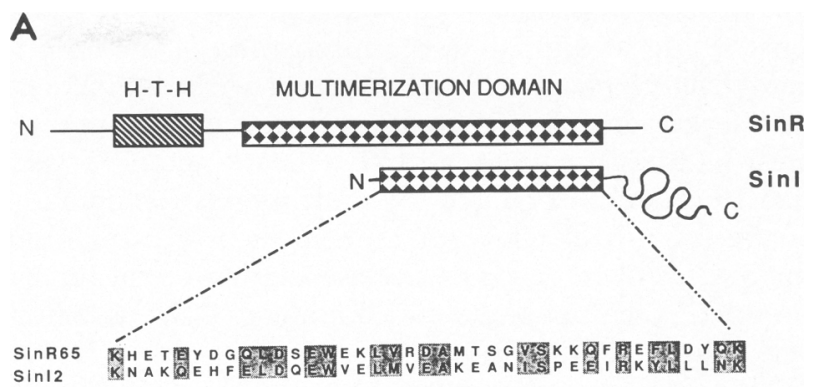

B
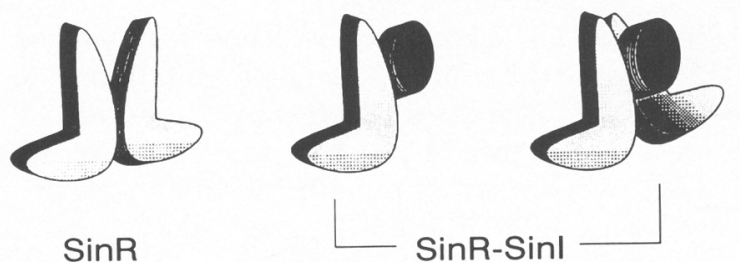

Figure 10. Proposed SinI/SinR interactions. $(A)$ A schematic model of SinI and SinR, including proposed functional domains, is shown at the top. The derived amino acid sequences for residues 65-104 of SinR and the SinI amino acid sequence from residue 2 to 40 shown below were aligned according to data base analysis. The shaded boxes indicate identical or chemically equivalent amino acids (Zuber et al. 1989). (B) Two possible models for SinI/SinR interaction are illustrated. The functional SinR multimer, which exists predominantly as a tetramer in solution (Gaur et al. 1991), is shown (left) as a dimer, for clarity. The DNA-binding domain is at the base of the structure. (Center) SinI forming a heterodimer with a SinR monomer, which prevents SinR function. (Right) SinI is shown binding to the multimerization domain of the SinR functional dimer, causing an undefined change in structure, which prevents DNA binding.

multimers. These complexes may be incapable of binding to DNA targets. In this way SinI would act as a dominant-negative inhibitor of SinR function. Many systems in eukaryotic organisms exhibit this type of regulation. Id, a helix-loop-helix (HLH) protein, is missing the basic region adjacent to the HLH domain that is essential for the specific DNA binding in another HLH protein, MyoD (Benezra et al. 1990). Id associates with MyoD and prevents it from binding to DNA, as MyoD can no longer form homodimeric or heterodimeric complexes with other positively acting HLH proteins. I-POU, a POU domain protein in Drosophila, prevents the binding of the neuron-specific DNA regulatory POU protein Cf- 1 to its target DNA by forming a stable heterodimer (Treacy et al. 1991). Because I-POU is lacking two essential amino acids in the basic region, it cannot bind to DNA. A last example is IP-1. Although its genetic determinant has not been sequenced yet, IP-1 inhibits the DNA-binding activity of AP-1 (Jun) in mammalian cells by binding to the LZ region of AP-1 (Auwerx and Sassone-Corsi 1991) and is believed to function in a manner similar to Id and I-POU. It is also possible that SinI interacts with a func- 
tional SinR multimer to prevent its binding to DNA. This would be similar to the interaction of the Salmonella bacteriophage P22 antirepressor with the P22 and $\lambda \mathrm{Cl}$ repressors. The antirepressor prevents the two repressors from functioning in vivo /Susskind and Youderian 1983), and it binds to the multimerization domains of the functional repressor multimers in vitro. Unlike the $\operatorname{Sin} /$ SinR interaction, the association of antirepressor to $\lambda \mathrm{Cl}$ does not prevent its binding to DNA /R. Sauer, pers. comm.).

However, these theories of SinI/SinR interactions are purely speculative at this time. For example, the putative LZ region of SinR (amino acids 47-61) is not included in the area of similarity to SinI, which beings at amino acid 66. Therefore, evidence for the proposed model awaits the results of detailed mutational analysis of both proteins and SinR DNA target sites. In any case, the results presented here show that the novel interactions of two regulatory proteins not only influence cell growth and differentiation but may also allow for the coordination of different developmental pathways.

Interestingly, the activities of $\sigma^{\mathrm{B}}$ and $\sigma^{\mathrm{F}}$, two $\sigma$-factors that also control late growth processes in $B$. subtilis, are regulated post-translationally by proteins that are coordinately produced. $\sigma^{\mathrm{F}}$, the product of the spoIIAC gene, is negatively controlled by the product of the cotranscribed spoIIAB (Schmidt et al. 1990), and $\sigma^{\mathrm{B}}$, the product of the $\operatorname{sig} B$ gene, is negatively modulated by the product of the cotranscribed sig $B$-orf $W$ gene (Benson and Haldenwang 1992; Boylan et al. 1992). The nature of these interactions is not yet known but, as is the case with SinR/ SinI, is presumably the result of direct protein-protein binding. Thus, it seems that protein-protein interaction is not an uncommon feature of prokaryotic gene regulation, as is true for eukaryotic systems.

\section{Materials and methods}

\section{Plasmid and strain constructions}

All bacterial strains are derivatives of IS75 (leuA8 metB5 hisH2) unless otherwise indicated. pIS229 is a multicopy plasmid containing the $\sin R$ gene and a phleomycin resistance marker (Mandic-Mulec et al. 1992). pIS253 is derived from pBD347 (Weinrauch et al. 1990), with its $\mathrm{MboI}$ site inactivated by mung bean nuclease treatment. The region of sinI, spanning from $\mathrm{XbaI}$ to NruI, was amplified by polymerase chain reaction (PCR) (Perkin-Elmer Cetus, Norwalk, CT) from plasmid DNA of pIS74 (Gaur et al. 1986), also adding HindIII sites to the ends of the DNA fragment. The amplified DNA was restricted with HindIII and cloned in pIS253, resulting in pIS256. This plasmid was then transformed into IS75 $\left(\mathrm{Spo}^{+}\right)$, selecting for $\mathrm{Cm}^{\mathrm{r}}$. One of the transformants with a rough phenotype was saved as IS1576. pIS257 was constructed from pIS256 by digesting this plasmid DNA at its unique $M b o I-A v a I I$ sites. After filling-in and ligation reactions, this resulted in an in-frame deletion of $99 \mathrm{bp}$ in the $\sin I$ ORF. This deletion was then transferred into the chromosome by gene conversion, in IS75, as it was predicted that disruption of the chromosomal $\sin I$ should give a sporulationdeficient phenotype. One of the sporulation-deficient colonies, having this in-frame deletion, was saved as IS874. The DNA sequence of $\sin I$ in IS874 was determined (using the Sequenase version 2.0 DNA Sequencing Kit, U.S. Biochemical Corporation, Cleveland, $\mathrm{OH}$ ) to ensure that the deletion was in-frame and no other mutation was picked up during amplification. For complementation experiments, the HindIII fragment of sinI in pIS256 was cloned into the integration vector pCP115 (Price and Doi 1985), resulting in pIS259. IS791 contains an integrated sinI behind a pSPAC promoter. To construct this strain, the region containing sinI, flanked by HindIII and Sall, was amplified by PCR and cloned into pDR66 (obtained from Alan Grossman, Massachusetts Institute of Technology, Cambridge). pDR66 contains the lacI gene and the pSPAC promoter in a plasmid that replicates in E. coli and has an ampicillin resistance (Amp ${ }^{\mathrm{r}}$ ) gene and a chloramphenicol resistance $\left(\mathrm{Cm}^{\mathrm{r}}\right)$ gene that expresses in $B$. subtilis. This plasmid can integrate at the ectopic amy $E$ locus. After ligation, the resulting plasmid was linearized at the unique $S c a I$ site and was integrated into the $B$. subtilis chromosome at the amyE locus, selecting for $\mathrm{Cm}^{\mathrm{r}}$ and giving strain IS791. IS803 contains a comG-lacZ fusion and a kanamycin resistance marker integrated at the $a m y E$ locus and was obtained from David Dubnau (Public Health Research Institute, NY). IS 1593 and IS1594 were constructed by transforming IS803 with DNA from pIS256 and pIS257, respectively.

IS819 is a wild-type strain containing spoIIA-lacZ integrated into the chromosome. The spoIIA-lacZ fusion, which results from a Campbell-type insertion at the spoIIA locus, was described previously (Mandic-Mulec et al. 1992). IS820 was obtained by transforming IS819 with chromosomal DNA from IS791. IS879 was obtained by transforming IS874 with chromosomal DNA from IS819. IS657, used for protease assays, has spoIIG-lacZ integrated into the homologous site in the chromosome. IS 880 was obtained by transforming IS 657 with chromosomal DNA from IS874. IS1491 was constructed by transforming pIS229 DNA into IS657.

\section{Media and $\beta$-galactosidase determinations}

All media (NSM, Luria broth (LB), minimal salts competence media] were described previously (Weinrauch et al. 1990). $\beta$-Galactosidase determinations were performed as described previously (Mandic-Mulec et al. 1992).

\section{Photomicroscopy}

Samples from isogenic $B$. subtilis cultures grown overnight at $32^{\circ} \mathrm{C}$ in NSM or treated with IPTG for pSPAC constructs were spotted on microscopic slides. These were observed and photographed in a Nikon Diaphot TMD-EF2 microscope with phasecontrast optics.

\section{SDS-PAGE}

Proteins were analyzed by electrophoresis in polyacrylamide gel containing SDS, as described by Laemmli (1970), followed by Coomassie staining.

\section{Western blot analysis}

Samples were subjected to electrophoresis as described above, and the proteins were electroblotted to a sheet of nitrocellulose transfer membrane (Millipore Corp., Bedford, MA). SinI antibodies were raised in guinea pigs against a synthetic polypeptide containing amino acids $12-47$ of the SinI ORF. The SinR antibodies were raised in guinea pigs against purified $\operatorname{Sin} R$ protein. Blotting and immunotechniques were performed as described previously (Bai et al. 1990). 


\section{Overproduction and purification of SinI}

We cloned the HindIII fragment of pIS256, containing the sinI gene, in pT7-I (U.S. Biochemical) resulting in pIS281. After establishing this plasmid in E. coli BL21(DE3), a strain containing pLysS (Studier et al. 1990), these cells were grown in LB containing chloramphenicol $(20 \mu \mathrm{g} / \mathrm{ml})$ and ampicillin $(100 \mu \mathrm{g} / \mathrm{ml})$. When the culture reached $\mathrm{OD}_{600}$ of $0.6-1.0$, it was induced with $0.4 \mathrm{~mm}$ IPTG and grown for $2.5 \mathrm{hr}$. All subsequent steps were carried out at $4^{\circ} \mathrm{C}$. The cells were harvested and broken in a French pressure cell in a buffer containing $10 \mathrm{mM}$ Tris- $\mathrm{HCl} / \mathrm{pH}$ 8.2 ), $10 \mathrm{mM} \mathrm{MgCl}_{2}, 1 \mathrm{~mm}$ EDTA, $0.3 \mathrm{~mm}$ dithiothreitol (DTT), $5 \%$ glycerol, and $1 \mathrm{mM}$ PMSF, at $13,000 \mathrm{lb} / \mathrm{in}^{2}$. The lysate was centrifuged at $38,000 \mathrm{~g}$ for $40 \mathrm{~min}$ in a Sorvall centrifuge. An amount of the supernatant $(2.5 \mathrm{ml})$ equivalent to $75 \mathrm{mg}$ of protein was loaded onto a 8-ml DEAE-Sephacel (Sigma Chemical Co., St.Louis, MO) column, previously equilibrated with the same buffer. The column was washed with $40 \mathrm{ml}$ of the buffer and eluted with $30 \mathrm{ml}$ of $0-400 \mathrm{~mm}$ linear $\mathrm{KCl}$ gradient in the same buffer. The first few fractions contained almost pure SinI, detected by $12 \%$ SDS-PAGE and also by immunoanalysis as described above. Fractions containing SinI were pooled and stored at $-20^{\circ} \mathrm{C}$ in aliquots.

\section{Binding assays}

The binding of SinR to aprE was performed using the $3^{\prime}$ endlabeled 250-bp NotI-DdeI promoter fragment as described previously (Gaur et al. 1991). To test the effect of SinI on SinR binding to aprE, SinI and SinR were mixed and incubated for 15 min at room temperature, before adding the DNA probe. Incubation in the presence of DNA was continued for $15 \mathrm{~min}$ more, and the products were separated on a $5 \%$ nondenaturing polyacrylamide gel at room temperature.

\section{Immunoprecipitation experiments}

Immunoprecipitation experiments were performed as described elsewhere (Bengal et al. 1992), with the modification presented below. $\left[{ }^{35} \mathrm{~S}\right]$ Methionine-labeled SinI was synthesized in vitro using an E. coli S-30 lysate (Amersham), supplemented with $\mathrm{T}_{7}$ RNA polymerase, under conditions suggested by the manufacturer. Lysate samples containing labeled SinI were incubated with purified Sin protein at room temperature for $30 \mathrm{~min}$ in a buffer containing $20 \mathrm{~mm}$ HEPES-KOH (pH 7.9), $50 \mathrm{mM} \mathrm{KCl}, 2.5$ $\mathrm{mM} \mathrm{MgCl}_{2}, 1 \mathrm{~mm}$ DTT, and $10 \%$ glycerol. The SinI/SinR complexes were then diluted to $200 \mu \mathrm{l}$ with RIPA buffer, which contained $10 \mathrm{~mm}$ Tris- $\mathrm{HCl}$ (pH 7.5), $150 \mathrm{~mm} \mathrm{NaCl}, 2 \mathrm{~mm}$ EDTA, $0.5 \% \mathrm{NP}-40$, and $1 \mathrm{mM}$ PMSF. SinR antibodies raised against purified SinR protein were then added. After overnight incubation at $4^{\circ} \mathrm{C}$, protein A-Sepharose beads $(40 \mu \mathrm{l})$ in RIPA buffer were added to the immune complexes, and they were placed in a rotary shaker for $2 \mathrm{hr}$ at $4^{\circ} \mathrm{C}$. After washing three times with RIPA buffer, the immune complexes were reduced by boiling in $2 \times$ SDS-PAGE sample buffer; they were then analyzed by SDSPAGE, followed by autoradiography. For coimmunoprecipitation studies with nonradioactive proteins, $2 \mu \mathrm{g}$ of purified SinR was mixed with $4 \mu \mathrm{g}$ of purified SinI and incubated at room temperature for $30 \mathrm{~min}$. Immunoprecipitation with SinI-specific antisera was done as described for SinR antisera above. The immunocomplex was electrophoresed on a 15\% SDS-polyacrylamide gel, transferred to nitrocellulose, and probed with SinRspecific antisera, as described previously (Bai et al. 1990).

\section{Protease assays}

The protease activity in culture supernatants was assayed as described previously (Gaur et al. 1986). Azocoll (25 mg) (Sigma
Chemical Co.) was added to suitable samples of cell supernatants in $0.1 \mathrm{M}$ Tris-hydrochloride $(\mathrm{pH} 7.5)$ and water to give a final volume of $5 \mathrm{ml}$. The reaction mixture was incubated at $37^{\circ} \mathrm{C}$ for $10 \mathrm{~min}$ with shaking. The digests were then filtered through Whatman filter paper, and the $\mathrm{OD}_{520}$ was measured in the filtrates. The blank was prepared the same way except that enzyme-free medium was added. Activity was defined as the change in $\mathrm{OD}_{520}$ by $1 \mathrm{ml}$ of culture supernatant at $37^{\circ} \mathrm{C}$ in 10 min (one optical density unit is equivalent to 1 unit of activity). The specific activity was obtained by dividing the activity by the corresponding number of Klett units of the culture.

\section{Acknowledgments}

We thank N. Gaur for originally suggesting that SinI might antagonize SinR activity. We appreciate discussions held with D. Dubnau and members of the Smith laboratory: L. Doukhan, G. Nair, M. Predich, and M. Zelic. We are especially grateful to $M$. Predich for his construction of a plasmid containing the sinI gene behind the pSPAC promoter and for the integration of this construct into the chromosome. We thank Guoling Tian and Werner Maas for providing the ArgR protein and DNA containing an Arg box. Special thanks go to Xueying Qiao for his advice and assistance. We thank Annabel Howard for expert secretarial assistance. This work was supported by U.S. Public Health Service grant GM32651 from the National Institutes of Health (NIH) awarded to I.S. U.B. and I.M.-M. were supported by U.S. Public Health Service training grant 5T32-AI67180 from the NIH awarded to the Department of Microbiology, New York University Medical Center. Computer analysis was performed on a Vax 11/750 purchased with funds from National Science Foundation grant PCM-831356, awarded to the Public Health Research Institute.

The publication costs of this article were defrayed in part by payment of page charges. This article must therefore be hereby marked "advertisement" in accordance with 18 USC section 1734 solely to indicate this fact.

\section{Note added in proof}

Another example of protein-protein interaction down-regulating the activity of a prokaryotic DNA-binding protein is found in E. coli bacteriophage $\mathrm{Pl}$. The Coi protein is an antagonist of $\mathrm{Cl}$ repressor function in vivo and in vitro [T. Heinzel et al. (1990) J. Biol. Chem. 265: 17928-17934].

The sequence data described in this paper have been submitted to the EMBL/GenBank data libraries under accession number M14112.

\section{References}

Albano, M., J. Hahn, and D. Dubnau. 1987. Expression of competence genes in Bacillus subtilis. J. Bacteriol. 169: 31103117.

Auwerx, J. and P. Sassone-Corsi. 1991. IP-1: A dominant inhibitor of Fos/Jun whose activity is modulated by phosphorylation. Cell 64: 983-993.

Bai, U., M. Lewandoski, E. Dubnau, and I. Smith. 1990. Temporal regulation of the Bacillus subtilis early sporulation gene spoOF. I. Bacteriol. 172: 5432-5439.

Benezra, R., R.L. Davis, D. Lockshon, D.L. Turner, and H. Weintraub. 1990. The protein Id: A negative regulator of helixloop-helix DNA binding proteins. Cell 61: 49-59.

Bengal, E., L. Ransone, R. Scharfmann, V.J. Dwarki, S.J. Tap- 
scott, H. Weintraub, and I.M. Verma. 1992. Functional antagonism between c-Jun and and MyoD proteins: A direct physical association. Cell 68: 507-519.

Benson, A.K. and W.G. Haldenwang. 1992. Characterization of a regulatory network that controls $\sigma^{\mathrm{B}}$ expression in Bacillus subtilis. J. Bacteriol. 174: 749-757.

Boylan, S.A., A. Rutherford, S.M. Thomas, and C.W. Price. 1992. Activation of Bacillus subtilis transcription factor $\sigma^{\mathrm{B}}$ by a regulatory pathway responsive to stationary-phase signals. $J$. Bacteriol. 174: 3695-3706.

Dubnau, E., J. Weir, G. Nair, L. Carter III, C. Moran Jr., and I. Smith. 1988. Bacillus sporulation gene spoOH codes for $\sigma^{30}$ $\left(\sigma^{\mathrm{H}}\right)$. I. Bacteriol. 170: 1054-1062.

Gaur, N.K., E. Dubnau, and I. Smith. 1986. Characterization of a cloned Bacillus subtilis gene that inhibits sporulation in multiple copies. J. Bacteriol. 168: 860-869.

Gaur, N.K., K. Cabane, and I. Smith. 1988. Structure and expression of the Bacillus subtilis sin operon. J. Bacteriol. 170: 1046-1053.

Gaur, N.K., J. Oppenheim, and I. Smith. 1991. The Bacillus subtilis sin gene, a regulator of alternate developmental processes, codes for a DNA-binding protein. I. Bacteriol. 173: 678-686.

Laemmli, U.K. 1970. Cleavage of structural proteins during the assembly of the head of bacteriophage T4. Nature 227: 680685.

Mandic-Mulec, I., N. Gaur, U. Bai, and I. Smith. 1992. Sin, a stage-specific repressor of cellular differentiation. I. Bacteriol. 174: 3561-3569.

Predich, M., G. Nair, and I. Smith. 1992. Bacillus subtilis early sporulation genes kiinA, spoOF, and spoOA are transcribed by the RNA polymerase containing $\sigma^{\mathrm{H}}$. J. Bacteriol. 174: $2771-2778$

Price, C.W. and R.H. Doi. 1985. Genetic mapping of $r p o D$ implicates the major sigma factor of Bacillus subtilis RNA polymerase in catabolite control of sporulation. Mol. \& Gen. Genet. 201: 88-95.

Schmidt, R., P. Margolis, L. Duncan, R. Coppolecchia, C.P. Moran Jr., and R. Losick. 1990. Control of developmental transcription factor $\sigma^{\mathrm{F}}$ by sporulation regulatory proteins SpoIIAA and SpoIIAB in Bacillus subtilis. Proc. Natl. Acad. Sci. 87: 9221-9225.

Sekiguchi, J., B. Ezaki, K. Kodama, and T. Akamatsu. 1988. Molecular cloning of a gene affecting the autolysin level and flagellation in Bacillus subtilis. J. Gen. Microbiol. 134: $1611-1621$

Smith, I. 1989. Initiation of sporulation. In Regulation of procaryotic development (ed. I. Smith, R. Slepecky, and P. Setlow), pp. 185-210. American Society for Microbiology, Washington, D.C.

1993. Pleiotropic regulatory proteins which control late growth development in Bacillus subtilis. In Bacillus subtilis and other gram-positive bacteria: Physiology, biochemistry and molecular biology (ed. A.L. Sonenshein, R. Losick, and J.A. Hoch). American Society for Microbiology, Washington, D.C.

Smith, I., I. Mandic-Mulec, and N. Gaur. 1991. The role of negative control in sporulation. Res. Microbiol. 142: 831-839.

Studier, F.W., A.H. Rosenberg, J.J. Dunn, and J.W. Dubendorff. 1990. Use of T7 RNA polymerase to direct the expression of cloned genes. Methods Enzymol. 185: 60-89.

Susskind, M.M. and P. Youderian. 1983. Bacteriophage P22 antirepressor and its control. In Lambda $I$ / ed. R.W. Hendrix, J.W. Roberts, F.W. Stahl, and R.A. Weisberg), pp. 347-363. Cold Spring Harbor Laboratory, Cold Spring Harbor, New York.
Trach, K., D. Burbulys, M. Strauch, J. Wu, N. Dhillon, R. Jonas, C. Hanstein, P. Kallio, M. Perego, T. Bird, G. Spiegelman, C. Fogher, and J.A. Hoch. 1992. Control of the initiation of sporulation in Bacillus subtilis by a phosphorelay. Res. Microbiol. 142: 815-823.

Treacy, M.N., X. He, and M.G. Rosenfeld. 1991. I-POU: A POUdomain protein that inhibits neuron-specific gene activation. Nature 350: $577-584$

Van Kaer, L., Y. Gansemans, M. Van Montagu, and P. Dhaese. 1988. Interaction of the Bacillus subtilis phase $\$ 105$ repressor with operator DNA: A genetic analysis. EMBO I. 7: 859866.

Weinrauch, Y., R. Penchev, E. Dubnau, I. Smith, and D. Dubnau. 1990. A Bacillus subtilis regulatory gene product for genetic competence and sporulation resembles sensor protein members of the bacterial two-component signal-transduction systems. Genes \& Dev. 4: 860-872.

Wu, J., P.J. Piggot, K.M. Tatti, and C.P. Moran Jr. 1991. Transcription of the Bacillus subtilis spoIIA locus. Gene 101: 113-116.

Zuber, P., J. Healy, H.L. Carter III, S. Cutting, C.P. Moran Jr., and R. Losick. 1989. Mutation changing the specificity of an RNA polymerase sigma factor. I. Mol. Biol. 206: 605-614. 


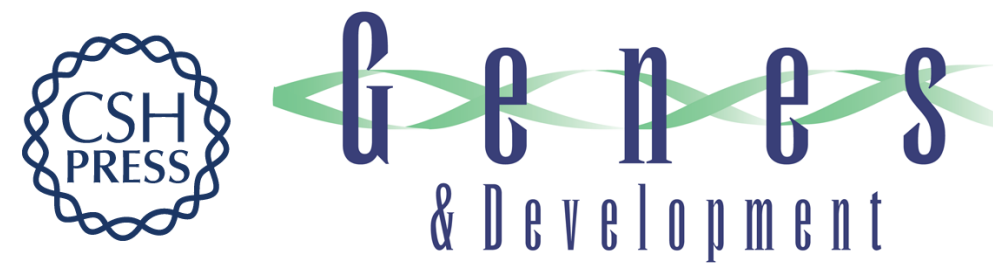

\section{Sinl modulates the activity of SinR, a developmental switch protein of Bacillus subtilis, by protein-protein interaction.}

U Bai, I Mandic-Mulec and I Smith

Genes Dev. 1993, 7:

Access the most recent version at doi:10.1101/gad.7.1.139

References This article cites 25 articles, 12 of which can be accessed free at:

http://genesdev.cshlp.org/content/7/1/139.full.html\#ref-list-1

License

Email Alerting

Service

Receive free email alerts when new articles cite this article - sign up in the box at the top right corner of the article or click here.

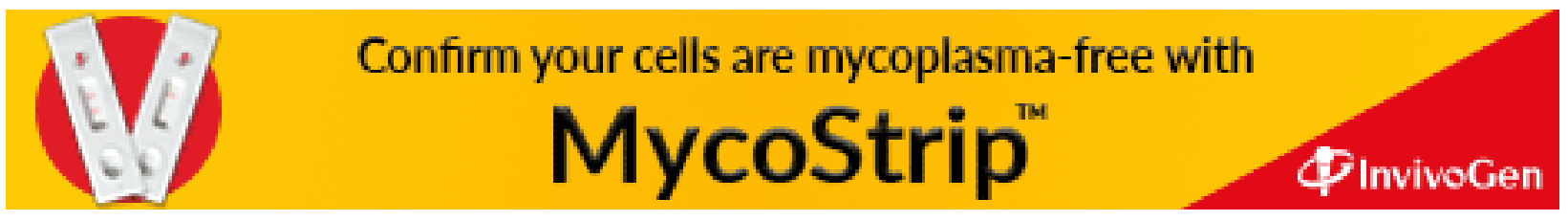

